\title{
Errors of UAV Autonomous Landing System for Different Radio Beacon Configurations
}

\author{
J.M. Kelner \& C. Ziółkowski \\ Military University of Technology, Warsaw, Poland
}

\begin{abstract}
At the turn of the 20th and 21st centuries, development of microelectronics and microwave techniques allowed for minimization of electronic devices and systems, and the use of microwave frequency bands for modern radio communication systems. On the other hand, the global navigation satellite system (GNSS) have contributed to the popularization of radio navigation in civilian applications. These factors had a direct impact on the development and dissemination of unmanned aerial vehicles (UAVs). In the initial period, the UAVs were used mainly for the army needs. This results also from the legal aspects of the UAV use in the airspace. Currently, commercial UAVs for civilian applications, such as image recognition, monitoring, transport, etc., are presented increasingly. Generally, the GNSS system accuracy for the UAV positioning during a flight is enough. However, the GNSS use for automatic takeoff and landing may be insufficient. The extensive, ground-based navigation support systems used at airports by manned aircraft testify to these. In the UAV case, such systems are not used due to their complexity and price. For this reason, the novel dedicated take-off and landing systems are developed. The proposal of the autonomous landing system, which is based on the Doppler effect, was presented in 2017. In this case, the square-based beacon configuration was analyzed. This paper shows the influence of various beacon configurations in the Doppler-based landing system on the positioning error during the UAV landing approach.
\end{abstract}

\section{INTRODUCTION}

At the end of the 20th century, the dynamic development of microelectronics caused the popularization of unmanned aerial vehicles (UAVs), also called drones. The UAV is an aircraft that does not require crew on-board and is unable to take passengers. The UAV can be remote control by a human operator or autonomously by on-board computers. Hence, the idea of the UAV has its roots in the second half of the last century, when the remotecontrolled models of aircraft, cars, or ships were mainly of a hobby nature. The V-1 flying bomb and V2 rocket from the 2nd World War can be regarded as the UAV prototypes. In the post-war period, research into the development of this technology for the army needs was conducted mainly in the USA and USSR. This development was directly related to the conquest of the cosmos in which unmanned spacecrafts, i.a., artificial satellites, were used.

Initially, due to legal restrictions, the UAVs were used mainly in the armed forces [1-3]. Originally, their main area of applications was image, optical, and radar recognition. These are so-called surveillance UAVs, e.g., the Northrop Grumman RQ4 Global Hawk. Then, the UAVs were also used to transposing weapons. This kind is called as a unmanned combat aerial vehicle (UCAV), e.g., the General Atomics MQ-9 Reaper also called the 
Predator B. Currently, the UAVs are widely used on the civilian market. Many private companies provide various services using the UAVs, e.g., in the field of energetics [4-7], agriculture [8,9], forestry and fire detection [10,11], water management [12] and flood detection [13], environmental protection [14], search and rescue [15], radio-communication [16], transport [17], etc.

The growth of the UAV market has also contributed to the development of other unmanned platforms, such as unmanned ground (UGVs), surface (USVs), and underwater vehicles (UUVs). The main recipients of this market sector are still the armed forces of various countries. In a report [18], the European Commission indicates the importance of this technology in the economic and technological development of countries, especially in the civilian sector [19]. According to the presented forecasts, the estimated value of the UAV market in 2019 will be expected to reach around 11-12 billion dollars. In 2015 and 2016, the domestic market was estimated at 165 and 200 million Polish zlotys, respectively [20].

In literature, we can find synonymous for the UAV such as unmanned aerial system (UAS) or remotely piloted aircraft systems (RPAS). Sometimes, the differences between the UAV and UAS are indicated. Then, the UAV is referred to itself aircraft platform, while the UAS includes also other system components, such as the ground-based flight control system. In this case, the RPAS is a synonym of the UAS. Currently, the RPAS is more widely used in military terminology, especially in the North Atlantic Treaty Organization (NATO) and European Defense Agency (EDA). In the literature, various classifications of the UAVs are presented. They consider different technical aspects or applications. From the viewpoint of this paper, the UAV term is referenced to a vertical take-off and landing (VTOL) aircraft, unlike a conventional take-off and landing (CTOL) aircraft requiring a runway. In the remainder of the paper, the VTOL is considered as a synonym of the VTOL UAV.

Global navigation satellite systems (GNSSs) [21,22] are commonly used in UAV navigation. In addition, remote control and video transmission from the aircraft to the remote UAV operator allows safe displacement of the drone. However, this solution may not be sufficient to take-off and landing approach. This is a significant problem, notably for the autonomous UAVs. The take-off and landing are the aircraft flight stages that require special precision in steering and navigation. For manned aircraft, the pilot on-board has more control over the plane or helicopter. On the other hand, dedicated take-off and approach systems are used at large civil and military airports. The instrument landing system (ILS), tactical air navigation system (TACAN) [23], or European Geostationary Navigation Overlay Service (EGNOS) $[21,22]$ are examples of radio local-area augmentation systems (LAASs). They are very important in bad weather conditions with limited visibility, e.g., fog, snowfall, or rain. Generally, the LAASs are not available to UAV majority. Therefore, it is important to develop such solutions, especially for autonomous drones.
In 2016, we proposed a landing system on a vessel for the manned and unmanned VTOL [24]. This system involves the use of terrestrial radio-beacons (RBs) and a dedicated navigation receiver (NR) placed onboard aircraft. In this case, the signal Doppler frequency (SDF) location method [25-27] is used to estimate the aircraft position relative to a landing pad on the ship. This solution [24] is based on the SDF applications dedicated to in-flight navigation and CTOL landing approach, which are shown in [28] and [29], respectively. Here, the analyzed concept of the landing approach system for the unmanned VTOL is a system modification shown in [30]. The proposed solution has been developed for the landing pad in hard-to-reach places such as oil platforms, vessels, islands, or skyscraper roofs. In [30], we assumed that RBs are located based on a square. The purpose of this paper is to assess the impact of selected configurations of the RBs on the VTOL positioning accuracy.

The remainder of the paper is organized as follows. Section 2 describes the SDF-based autonomous landing approach system. Assumptions and simulation scenarios are presented in Section 3. In Section 4 , the obtained simulation results are shown. In this case, the positioning accuracy of the VTOL UAV for different RB configurations is analyzed. The summary is in the final part of the paper.

\section{AUTONOMOUS LANDING APPROACH SYSTEM FOR VTOL UAV}

The spatial structure of the SDF-based autonomous landing approach system for the VTOL is shown in Figure 1.

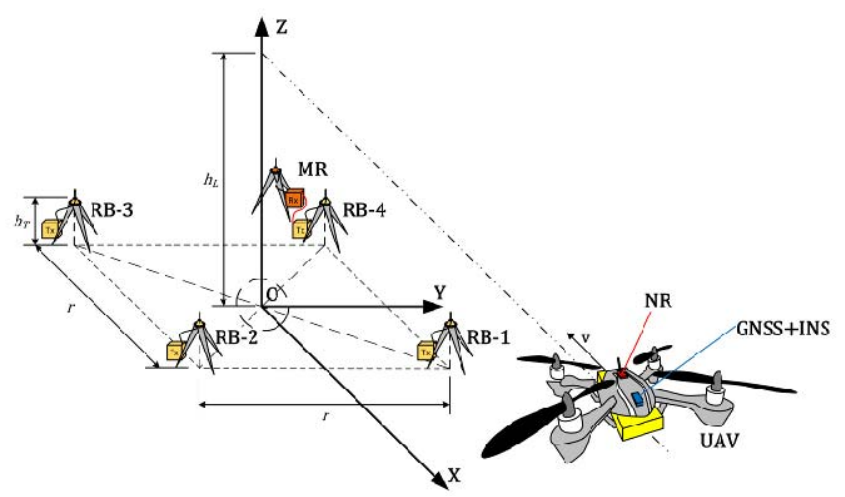

Figure 1. Spatial structure of autonomous landing approach system for VTOL [30]

The ground part of the system consists of four RBs and a measuring receiver (MR). In the solution discussed in [30], we assumed that four RBs are located based on the square around the landing pad. Each RB is equipped with a signal generator, power amplifier, and transmitting antenna placed at the stand. Additionally, the RB can be equipped with a rubidium or cesium frequency standard that will increase the frequency stability of transmitted signals. This is important from the viewpoint of the SDF used [31]. Three RBs, i.e., RB-1, RB-2, and RB-3, transmit harmonic signals at defined frequencies $f_{1}, f_{2}$, and $f_{3}$, respectively. At frequency $f_{4}$, the RB-4 transmits a 
modulated signal using differential phase shift keying (DPSK). In each transmitted frame, information about the location coordinates of the individual RBs and their frequency corrections are sent. These corrections are determined based on local measurements carried out by the MR located near RB-4.

The NRs placed on the VTOLs are the receiving part of the system. Each VTOL is equipped with typical elements of the navigation system, namely a GNSS receiver and inertial navigation system (INS). This allows to carry out a controlled or autonomous UAV flight phase. Whereas, the NR provides positioning the VTOL near the landing pad and its landing approach. The NR is tuned to the frequency band on which operate the RBs. This means that the NR operation band includes the carrier frequencies $f_{1}$, $f_{2}, f_{3}$, and the modulated-signal band at the frequency $f_{4}$. The NR is made in software-defined radio (SDR) technology [32,33]. This means that the NR provides signal processing and determining the estimated positions of the UAV relative to the landing pad. For each RB, the Doppler frequency shift (DFS) is determined every specified time-period $\Delta T$ based on the received signal with the duration of $T$ s. Then, the UAV coordinates are determined based on discrete instantaneous DFSs aggregated in a time window $T_{A}$. The method of the DFS determination for the harmonic signal is presented in [25-27]. In the case of the modulated signal from RB-4, after sub-band filtering, information frames are demodulated and the instantaneous DFSs are estimated based on a methodology shown in [34]. A detailed description of the autonomous landing approach system and estimating the VTOL coordinates based on the SDF is contained in [30].

The presented system can be classified as precise short-range radio navigation systems. It can be used during the UAV landing approach, as well as its takeoff from the landing field and in-flight in an area, where is a radio-range between the NR and RBs. A key advantage of the proposed solution is its narrow band with relatively high positioning precision. In the case of systems based on time measurement, e.g., $[35,36]$, obtaining comparable accuracy would require the use of a much wider band. We point out that the frequency allocation for this type of dedicated system is a serious problem. For the developed system, unlicensed frequency bands, e.g., dedicated to the WiFi included in industrial, scientific and medical (ISM) bands, can be used for this purpose. In this case, the emission of the harmonic signals with more power than the emission average in the band does not constitute a significant interference to other systems.

\section{SCENARIO AND ASSUMPTIONS FOR SIMULATION STUDIES}

In a scenario shown in [30], we assumed that the RBs are placed on the basis of the square with a side length equal $r$ (see Figure 1 ). In this paper, we analyze three configurations of the RB positions based on other regular polygons, i.e. a triangle, pentagon, and hexagon. A common feature of all configurations is the radius $\mathrm{R}$ of a circumscribed circle. Figure 2 presents the analyzed RB configurations together with a reference configuration based on the square (see Figure 2 (b)).
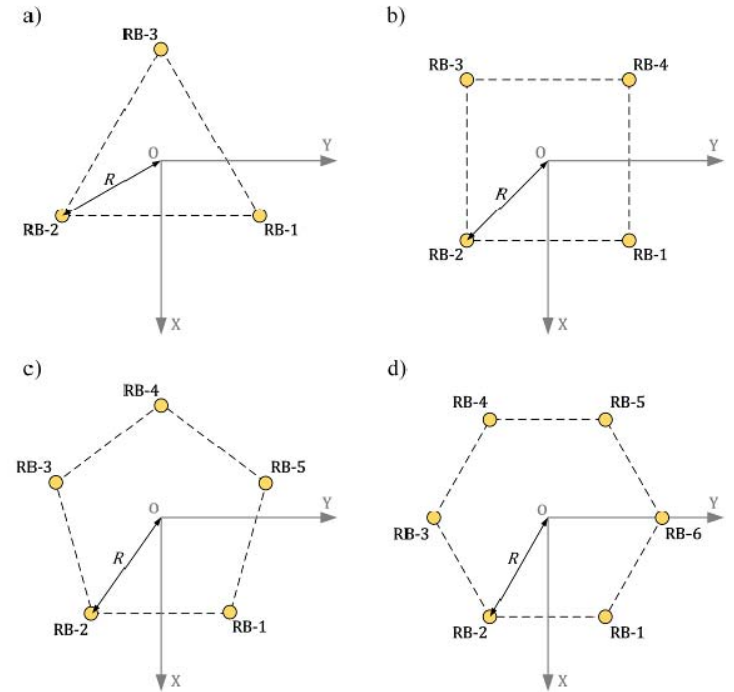

Figure 2. Analyzed spatial configurations of RBs based on regular polygons: a) triangle, b) rectangle, c) pentagon, d) hexagon

In addition, in simulation studies, we assume similar assumptions as in [30], i.e.,

- landing point at $\mathrm{O}$ is the origin of the local coordinate system;

- the system configuration based on the regular polygon consists of $K$ RBs, where $K=3,4,5,6$, for the regular triangle, rectangle (i.e., square), pentagon, and hexagon, respectively (see Figure 2);

- assuming the distance $r=40 \mathrm{~m}$ [30] between neighboring $\mathrm{RBs}$ in the square configuration, the radius $R \approx 28.3 \mathrm{~m}$ is a base for determining the $R B$ coordinates with respect to the point $\mathrm{O}$ in each configuration; the location coordinates of the individual RBs for the analyzed configurations are contained in Table 1; the height of the RB transmitting antennas is $h_{T}=z_{k}=2 \mathrm{~m}$ for $k=1, . ., K$;

- the system operates in the ISM band used by the Wi-Fi, i.e., 2.4 GHz; in each configuration, the $k$-th RB transmits the harmonic signal at $f_{k}$, where $k=1, . ., K-1$; while, the $K$-th RB emits the DPSK signal at $f_{K}$; these frequencies are determined as follows: $\quad f_{K}(\mathrm{kHz})=2399800+50 \cdot K+100 \quad$ and $f_{k}(\mathrm{kHz})=2399800+50 \cdot(k-1) \quad$ for $k=1, . ., K-1$; the bandwidth of the DPSK signal is equal to $B_{T}=80 \mathrm{kHz}$;

- the NR operates at the frequency $f_{R}=2.4 \mathrm{GHz}$ with the reception band $B_{R}=500 \mathrm{kHz}$;

for a Doppler curve (DFSs versus time) analysis, the time window $T_{A}=5.0 \mathrm{~s}$ is used;

- in an electromagnetic environment, an additive white Gaussian noise (AWGN) is occurred, and a level of the emitted signals at the farthest point $(\mathrm{L})$ of an analyzed trajectory is ensured by a signal-tonoise ratio equal to $S N R=8 \mathrm{~dB}$;

- the VTOL flight between the $\mathrm{L}$ and $\mathrm{P}$ points is carried out at a constant altitude $\mathrm{hL}=50 \mathrm{~m}$ with a velocity $\mathrm{v}=72 \mathrm{~km} / \mathrm{h}=20 \mathrm{~m} / \mathrm{s}$ (see Figure 3); then, the flight ceiling is lowered;

- the length of the analyzed VTOL flight route, i.e., the distance between the $\mathrm{L}$ and $\mathrm{P}$ points, is equal to $\mathrm{d}=400 \mathrm{~m}$. 
Table 1. Coordinates of RB positions on OXY plane for different configurations

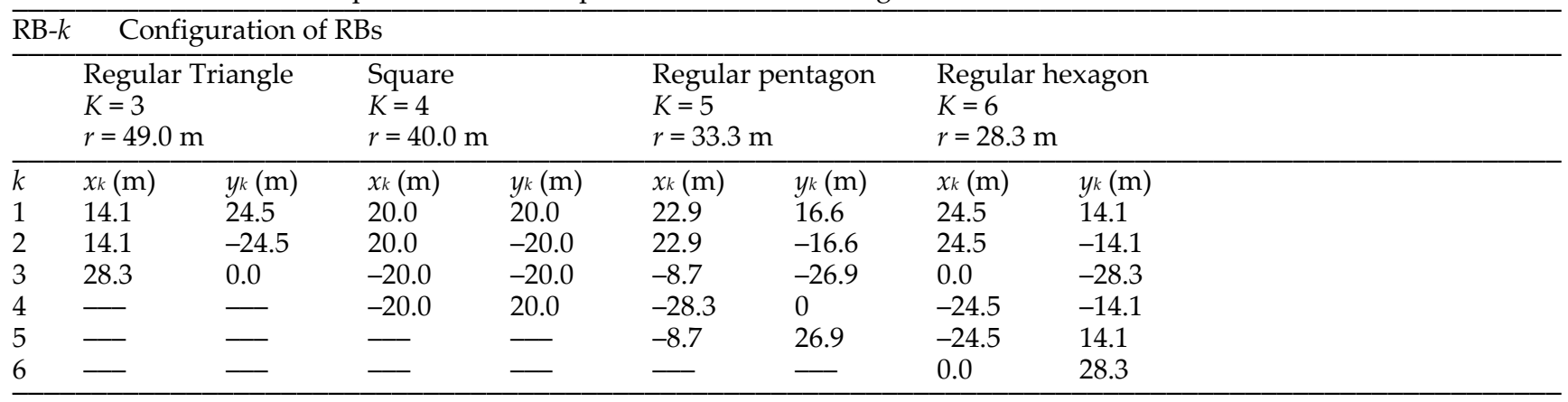

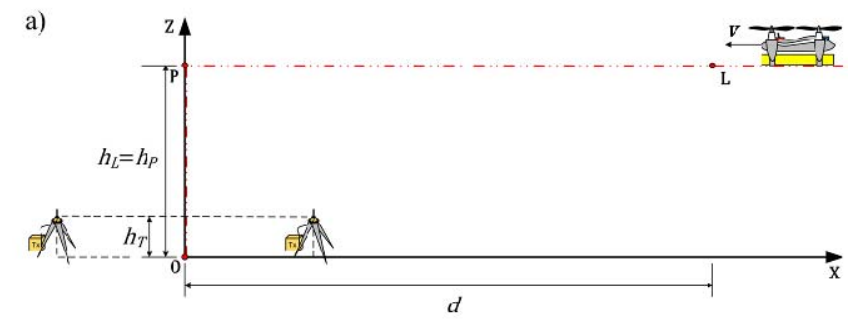

configurations. Additionally, the average errors shown by dashed lines.

a)

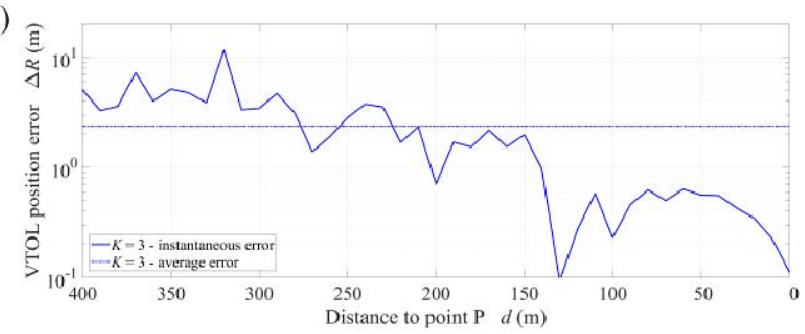

b)

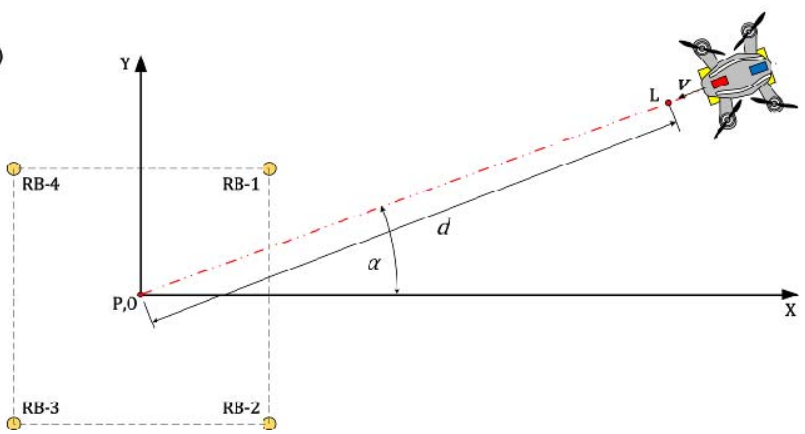

Figure 3. Spatial scenario of VTOL landing approach on example of $\mathrm{RB}$ reference configuration projected in plane: a) OXZ and b) OXY [30]

Simulation studies are carried out for the UAV movement trajectory depicted in Figure 3. In this case, two scenarios are considered. In the first scenario, Sc.1, we evaluate the VTOL position error along the LP trajectory for different RB configurations and the approach direction $\alpha=0$. In the second scenario, Sc.2, the VTOL positioning error is analyzed at the point $\mathrm{P}$ for the various $\alpha$ directions.

\section{RESULTS OF SIMULATION STUDIES}

For the assumptions presented in Section 3, we conducted simulation studies. In our analysis, the VTOL positioning error is a basic measure of the accuracy assessment of the developed navigation system. This measure is defined as follows

$$
\Delta R=\sqrt{\left(x-x_{0}\right)^{2}+\left(y-y_{0}\right)^{2}+\left(z-z_{0}\right)^{2}}
$$

where $\left(x_{0}, y_{0}, z_{0}\right)$ and $(x, y, z)=$ the real and estimated coordinates of the UAV position, respectively.

The simulation results obtained for Sc.1 are illustrated in Figure 4. In this case, graphs of the instantaneous positioning error for the VTOL landing approach are presented for four analyzed RB

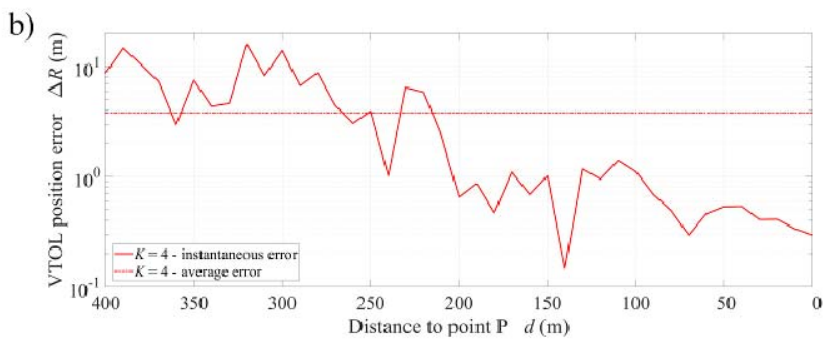

c)

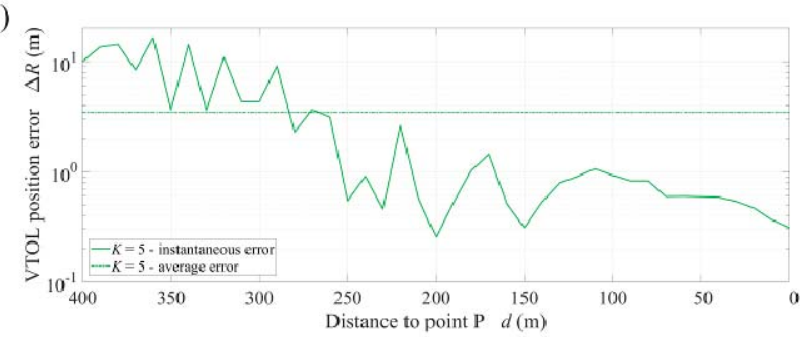

d)

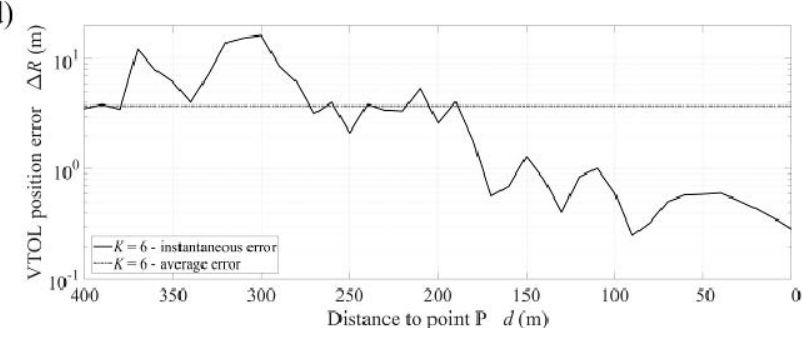

Figure 4. Instantaneous and average VTOL positioning errors versus distance $d$ to point $\mathrm{P}$ for various RBs configurations: a) $K=3$, b) $K=4$, c) $K=5$, and d) $K=6$

The obtained results show the high precision of the VTOL positioning based on the proposed system and SDF method. For $d<100 \mathrm{~m}$, the instantaneous error of the UAV position for each configuration is less than $1.0 \mathrm{~m}$. In the last second of approaching the point $\mathrm{P}$, the error is less than $0.5 \mathrm{~m}$. 
a)

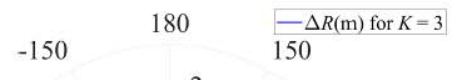

$$
-120
$$

$-90$

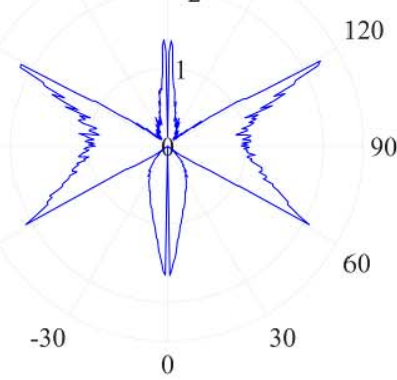

b)

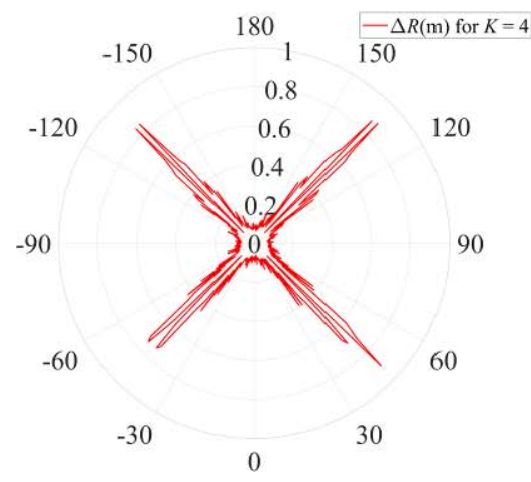

c)

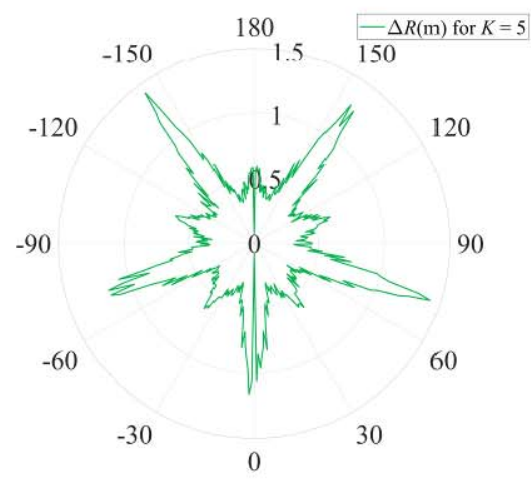

d)

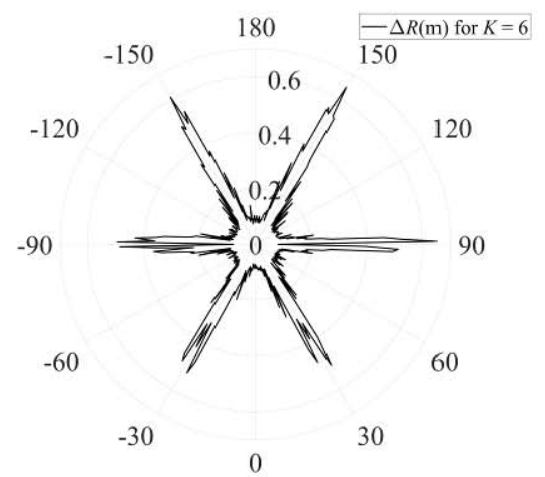

Figure 5. VTOL positioning error at point $\mathrm{P}$ versus approach direction $\alpha$ for different RB configurations: a) $K=3$, b) $K=4$, c) $K=5$, and d) $K=6$

In Sc.1, we may use the average error obtained on the entire analyzed route with the length of $400 \mathrm{~m}$ as a comparative measure. In this case, the average errors are equal to $2.4 \mathrm{~m}, 3.8 \mathrm{~m}, 3.4 \mathrm{~m}$, and $3.8 \mathrm{~m}$ for configurations based on the regular triangle, rectangle, pentagon, and hexagon, respectively. Therefore, we may conclude that the best results are obtained for $K=3$. This result may be related to the approach direction $\alpha=0$ assumed in Sc.1. Thus, for odd values of $K$, one of $\mathrm{RB}$ is in the direction of the UAV movement.
The impact of the VTOL approach direction relative to the adopted coordinate system is analyzed in Sc.2. The obtained simulation results are illustrated in Figure 5.

The obtained graph shapes of the UAV position error are closely related to the RB configurations depicted in Figure 2. In this case, the largest errors occur when the approach direction $\alpha$ coincides with the direction determined by the point $\mathrm{O}$ and the location of at least one RB. In the signal received from such RB, the estimated DFSs take maximum values and these data are not used in the SDF. This is particularly visible for $K=4$ and $K=6$, when a RB pair is always located in the analyzed directions.

The analysis of the results in Figure 5 shows that decreasing the number of RBs in the system does not necessarily lead to higher system accuracy. For comparison of the individual configurations, the errors at point $\mathrm{P}$ averaged over the approach direction are $0.76 \mathrm{~m}, 0.17 \mathrm{~m}, 0.55 \mathrm{~m}$, and $0.16 \mathrm{~m}$ for $K=3,4,5,6$, respectively. Therefore, this is the opposite case to that presented in Figure 4.

Generally, for each of the analyzed RB configurations, the VTOL positioning error at point $\mathrm{P}$ is always less than $2 \mathrm{~m}$ regardless of the direction $\alpha$. For most approach directions, the UAV position error is less than $0.5 \mathrm{~m}$. These values are very small in relation to the assumed radius of the landing pad equal to $R \approx 28.3 \mathrm{~m}$. Hence, we may conclude that the developed system allows the safe and autonomous landing approach even with sizable dimensions of the VTOL.

\section{CONCLUSION}

In this paper, we evaluate the influence of the RB configuration in the landing approach system for the VTOL UAV on its positioning error. The developed system is based on the DFS measurement in the signals received from the terrestrial RBs around the landing pad. The DFSs are measured in the dedicated NR, which is placed onboard aircraft. The SDF method is used to estimate the VTOL position relative to the landing site. Our analysis is based on simulation studies. In this case, we consider two scenarios and four RB configurations based on the regular polygons. The obtained result shows the high accuracy of the UAV positioning for all analyzed configurations. The best results at the point located above the landing center are obtained for the configuration consisting of six RBs. At this point and for this configuration, the mean error regardless of the approach direction was less than $20 \mathrm{~cm}$. The proposed solution seems ideal for use in stand-alone autonomous landing approach systems for the UAV. However, empirical research is still required, which is planned in the future.

The presented idea of the SDF-based navigation for UAVs can also be used for the needs of other types of autonomous vehicles. In the future, we consider using this concept to navigate autonomous USVs or manned vessels entering a port. In this case, the RBs will be located in the coastal zone around the port. 


\section{ACKNOWLEDGMENTS}

This work was developed within a framework of the Research Grant "Basic research in sensor technology field using innovative data processing methods" no. GBMON/13-996/2018/WAT sponsored by the Polish Ministry of Defense.

\section{REFERENCES}

[1] Groves, P.D. 2013. Principles of GNSS, inertial, and multisensor integrated navigation systems, 2nd ed. Boston, MA, USA: Artech House.

[1] Tortonesi, M., Stefanelli, C., Benvegnu, E., Ford, K., Suri, N. \& Linderman, M. 2012. Multiple-UAV coordination and communications in tactical edge networks. IEEE Communications Magazine 50(10): 48-55.

[2] Orfanus, D. de Freitas, E.P. \& Eliassen, F. 2016. Selforganization as a supporting paradigm for military UAV relay networks. IEEE Communications Letters 20(4): 804-807.

[3] Ma'sum, M.A., Arrofi, M.K., Jati, G., Arifin, F., Kurniawan, M.N., Mursanto, P. \& Jatmiko, W. 2013. Simulation of intelligent unmanned aerial vehicle (UAV) for military surveillance. 2013 International Conference on Advanced Computer Science and Information Systems (ICACSIS), Bali, Indonesia, 28-29 September 2013: 161-166.

[4] Babak, S., Myslovych, M. \& Sysak, R. 2016. Module structure of UAV-based computerized systems for remote environment monitoring of energy facilities. 2016 17th International Conference Computational Problems of Electrical Engineering (CPEE), Sandomierz, Poland, 1417 September 2016: 1-3.

[5] Daliento, S., Chouder, A., Guerriero, P., Pavan, A.M., Mellit, A., Moeini, R. \& Tricoli, P. 2017. Monitoring, diagnosis, and power forecasting for photovoltaic fields: A review. International Journal of Photoenergy 2017(e1356851): 1-13.

[6] Quater, P.B., Grimaccia, F., Leva, S. Mussetta, M. \& Aghaei, M. 2014. Light unmanned aerial vehicles (UAVs) for cooperative inspection of PV plants. IEEE Journal of Photovoltaics 4(4):1107-1113.

[7] Grimaccia, F., Aghaei, M., Mussetta, M., Leva, S. \& Quater, P.B. 2015. Planning for PV plant performance monitoring by means of unmanned aerial systems (UAS). International Journal of Energy and Environmental Engineering 6(1): 47-54.

[8] Berni, J.A.J., Zarco-Tejada, P.J., Suarez, L. \& Fereres, E. 2009. Thermal and narrowband multispectral remote sensing for vegetation monitoring from an unmanned aerial vehicle. IEEE Transactions on Geoscience and Remote Sensing 47(3): 722-738.

[9] Wijitdechakul, J., Sasaki, S., Kiyoki, Y. \& Koopipat, C. 2016. UAV-based multispectral image analysis system with semantic computing for agricultural health conditions monitoring and real-time management. 2016 International Electronics Symposium (IES), Denpasar, Indonesia, 29-30 September 2016: 459-464.

[10] Yuan, C., Liu, Z., \& Zhang, Y. 2016. Vision-based forest fire detection in aerial images for firefighting using UAVs. 2016 International Conference on Unmanned Aircraft Systems (ICUAS), Arlington, VA, USA, 7-10 June 2016: 1200-1205.

[11] Ghamry, K.A., Kamel, M.A. \& Zhang, Y. 2016. Cooperative forest monitoring and fire detection using a team of UAVs-UGVs. 2016 International Conference on Unmanned Aircraft Systems (ICUAS), Arlington, VA, USA, 7-10 June 2016: 1206-1211.

[12] Rathinam, S., $\quad$ Almeida, P., $\quad$ Kim, Z., Jackson, S., Tinka, A., Grossman, W. \& Sengupta, R. 2007. Autonomous searching and tracking of a river using an
UAV. 2007 American Control Conference (ACC), New York, NY, USA, 9-13 July 2007: 359-364.

[13] Popescu, D., Ichim, L. \& Caramihale, T. 2015. Flood areas detection based on UAV surveillance system. 2015 19th International Conference on System Theory, Control and Computing (ICSTCC), Cheile Gradistei, Romania, 14-16 October 2015: 753-758.

[14] Lu, Y. Macias, D. Dean, Z.S., Kreger, N.R. \& Wong, P.K. 2015. A UAV-mounted whole cell biosensor system for environmental monitoring applications. IEEE Transactions on NanoBioscience 14(8): 811-817.

[15] Erdos, D., Erdos, A. \& Watkins, S.E. 2013. An experimental UAV system for search and rescue challenge. IEEE Aerospace and Electronic Systems Magazine 28(5): 32-37.

[16] Mozaffari, M., Saad, W. Bennis, M. \& Debbah, M. 2017. Optimal transport theory for cell association in UAVenabled cellular networks. IEEE Communications Letters 21(9): 2053-2056.

[17] Hoareau, G., $\quad$ Liebenberg, J.J., $\quad$ Musial, J.G. \& Whitman, T.R. 2016. Package transport by unmanned aerial vehicles. Patent US20160068265 A1.

[18] Towards a European strategy for the development of civil applications of Remotely Piloted Aircraft Systems (RPAS). 2012. Brussels, Belgium: Council of the European Union.

[19] van Blyenburgh, P. 2012. Towards a European strategy for the development of civil applications of RPAS. UAS Vision. Available: https://www.uasvision.com/2012/09/07/towards-aeuropean-strategy-for-the-development-of-civilapplications-of-rpas/

[20] Duszczyk, M. 2017. Forecasts for the drone market are promising (in Polish). Rzeczpospolita, Available: https://www.rp.pl/Sylwetki/303209855-Prognozy-dlarynku-dronow-sa-obiecujace.html.

[21] Kaplan, E.D. \& Hegarty, C. (eds). 2005. Understanding GPS: Principles and applications, 2nd ed. Boston, MA, USA: Artech House, 2005.

[22] van Diggelen, F. 2009. A-GPS: Assisted GPS, GNSS, and SBAS. Boston, MA, USA: Artech House, 2009.

[23] Kayton, M. \& Fried, W.R. 1997. Avionics navigation systems, 2nd ed. New York, NY, USA: WileyInterscience.

[24] Kelner, J.M., Ziółkowski, C. \& Nowosielski, L. 2016. Local navigation system for VTOLs used on the vessels. 2016 IEEE/ION Position, Location and Navigation Symposium (PLANS), Savannah, GA, USA11-14 April 2016: 415-421.

[25] Kelner, J.M., Ziółkowski, C. \& Kachel, L. 2008. The empirical verification of the location method based on the doppler effect. 2008 17th International Conference on Microwaves, Radar and Wireless Communications (MIKON), Wrocław, Poland, 19-21 May 2008. vol. 3: 755758.

[26] Kelner, J.M. 2010. Analysis of the Doppler location method of the radio waves emission sources, Ph.D. Thesis (in Polish). Warsaw, Poland: Military University of Technology.

[27] Gajewski, P., Ziółkowski, C. \& Kelner, J.M. 2012. Using SDF method for simultaneous location of multiple radio transmitters. 2012 19th International Conference on Microwave Radar and Wireless Communications (MIKON), Warsaw, Poland, 21-23 May 2012. vol. 2: 634-637.

[28] Kelner, J.M. 2011. Positioning an aircraft using the TDSDF method. Polish Journal of Environmental Studies 20(5A): 80-84.

[29] Kelner, J.M. \& Ziółkowski, C. 2011. The concept of Doppler Landing Approach Precision System (DOLAPS) Polish Journal of Environmental Studies 20(5A): 85-90.

[30] Kelner, J.M. \& Ziółkowski, C. 2017. Doppler effectbased automatic landing procedure for UAV in difficult access environments. Journal of Advanced Transportation 2017(e8092718): 1-9. 
[31] Kelner, J.M., Ziółkowski, C. \& Marszałek, P. 2016. Influence of the frequency stability on the emitter position in SDF method. 2016 17th International Conference on Military Communications and Information Systems (ICMCIS), Brussels, Belgium, 23-24 May 2016: 16.

[32] Grayver, E. 2012. Implementing software defined radio. New York, NY, USA: Springer.

[33] Mitola, J. 2000. Software radio architecture: objectoriented approaches to wireless systems engineering. New York, NY, USA: Wiley.
[34] Kelner, J.M. \& Ziółkowski, C. 2015. The use of SDF technology to BPSK and QPSK emission sources' location (in Polish). Przeglad Elektrotechniczny 91(3): 6165.

[35] Stefański, J. 2015. Asynchronous time difference of arrival (ATDOA) method. Pervasive and Mobile Computing 23: 80-88.

[36] Sadowski, J. \& Stefański, J. 2017. Asynchronous phaselocation system. Journal of Marine Engineering $\mathcal{E}$ Technology 16(4): 400-408. 